\title{
Hypogonadism as an additional indication for bariatric surgery in male morbid obesity?
}

\author{
Jinous Samavat, Enrico Facchiano', Marcello Lucchese', Gianni Forti, \\ Edoardo Mannucci ${ }^{2}$, Mario Maggi ${ }^{3}$ and Michaela Luconi
}

Endocrinology Unit, Department of Experimental and Clinical Biomedical Sciences, University of Florence, Florence, Italy, ${ }^{1}$ Bariatric and Metabolic Surgery, Careggi Hospital, ${ }^{2}$ Diabetes Agency, Azienda Ospedaliera-Universitaria Careggi, Florence, Italy and ${ }^{3}$ Sexual Medicine and Andrology Unit, Department of Experimental and Clinical Biomedical Sciences, University of Florence, Viale Pieraccini 6, 50139 Florence, Italy
Correspondence should be addressed to M Maggi

Email mario.maggi@unifi.it

\begin{abstract}
Objective: Male obesity is often associated with reduced levels of circulating total (TT) and calculated free testosterone (cFT), with normal/reduced gonadotropins. Bariatric surgery often improves sex steroid and sex hormone-binding globulin (SHBG) levels. The aim of this study was to assess the effects of bariatric surgery on waist circumference (WC) and BMI, and on TT levels, in morbidly obese men, stratified, according to the gonadal state, in eugonadal and hypogonadal $(\mathrm{TT}<8 \mathrm{nmol} / \mathrm{l})$ subjects. Design: A cohort of morbidly obese patients ( 29 with hypogonadism (HG) and 26 without) undergoing bariatric surgery (37, 10, 6, and 2, with Roux-en-Y gastric bypass, laparoscopic adjustable gastric banding, biliopancreatic diversion and gastric sleeve, respectively) was studied at 6 and 12 months from the operation.

Methods: Anthropometric parameters (weight, BMI, WC) and sex hormones (gonadotropins, TT, cFT, estradiol $\left(\mathrm{E}_{2}\right.$ ), SHBG) were assessed.

Results: WC was the only parameter significantly correlated with androgens, but not with $E_{2}$, SHBG, and gonadotropins, at baseline. After surgery, a significant increase in TT, CFT, and SHBG, accompanied by a decrease in $\mathrm{E}_{2}$, was evident in the two groups. However, both TT and $\mathrm{CFT}$, but not $\mathrm{E}_{2}, \mathrm{SHBG}$, and gonadotropin variations, were significantly higher in the hypogonadal group at follow-up, with an overall $93 \%$ complete recovery from HG. Reduction in WC, but not BMI, was significantly greater in hypogonadal men $(\triangle \mathrm{WC}=-29.4 \pm 21.6$ vs $-14.4 \pm 17.4$ at 12 months, $P=0.047)$.

Conclusions: Recovery from obesity-associated HG is one of the beneficial effects of bariatric surgery in morbidly obese men. The present findings suggest that the gonadal state is a predictor of WC decrease after bariatric surgery.

\section{Introduction}

Obesity and its associated comorbidities have been exponentially increasing during the last 40 years, representing a medical and socio-economic burden, not only in industrialized, but also in developing countries (1). Treatments for obesity include a wide range of interventions, such as dietary prescriptions, structured exercise, lifestyle modification programs, specific drugs, and bariatric surgery. The currently recognized indications of bariatric surgery are limited to morbid obesity, i.e. BMI> $40 \mathrm{~kg} / \mathrm{m}^{2}$, or $\mathrm{BMI} \geq 35 \mathrm{~kg} / \mathrm{m}^{2}$ associated with risky comorbidities, such as cardiovascular (CV) diseases and type 2 diabetes (T2DM), which are significantly ameliorated after the intervention $(2,3,4)$.

Alterations in sex steroid hormones are often associated with male obesity. In particular, low circulating levels of total (TT) and free (FT) testosterone in the presence of reduced gonadotropins, characterizing central secondary hypogonadism (HG), with elevated estrogens, have been described in male metabolic syndrome $(5,6)$. Obesity and HG are linked in a vicious cycle, in which low testosterone favors weight gain, and adiposity induces $\operatorname{HG}(6,7)$. Interestingly, both obesity and HG are associated with
(C) 2014 European Society of Endocrinology Printed in Great Britain
Published by Bioscientifica Ltd. 
increased all-cause and CV mortality $(6,8)$. Several studies demonstrated that weight loss and reduction in waist circumference (WC) induce an increase in testosterone levels. A recent meta-analysis (9) comparing bariatric surgery with lifestyle modifications programs has shown a greater recovery in TT and FT with the surgical approach, probably due to a greater effect on body weight. On the other hand, it is possible that androgen levels, in men, contribute to the regulation of body weight. Hypogonadal subjects, who have reduced muscle mass and lower muscular strength, could also have a reduced energy expenditure as described for aging males (10); in addition, androgens could play a role in the regulation of adipose tissue metabolism (11).

The aim of this study was to verify whether hypogonadism (TT $<8 \mathrm{nmol} / \mathrm{l}$ ) modulates the effects of bariatric surgery on abdominal adiposity, body weight, and TT.

\section{Subjects and methods}

\section{Patients}

The clinic-based survey was performed on a consecutive series of morbidly obese $\left(\mathrm{BMI}>40 \mathrm{~kg} / \mathrm{m}^{2}\right)$ patients who were undergoing bariatric surgery at Careggi University Hospital in Florence $(n=55)$. All patients were enrolled for a subsequent 6- and 12-month follow-up, after intervention. The study was approved by the Local Ethical Committee and Institutional Review Board (protocol number 83/13). All patients enrolled gave signed informed consent after receiving written and oral information on the study.

\section{Study design}

All male patients in the list for bariatric surgery at the Metabolic and Bariatric Surgery Unit of Careggi
University Hospital in Florence, Italy, between July 1, 2009 and June 30, 2011, who gave their consent were enrolled in the study, provided that they had a BMI $>40 \mathrm{~kg} / \mathrm{m}^{2}$ and no history of present or past treatment for HG. The choice of surgery and type of surgery was made by a team composed of a surgeon, an endocrinologist, and a dietitian, depending on the overall evaluation of the patient's history. Among the 55 patients studied, 37, 10, 6, 2, underwent Roux-en-Y gastric bypass (67\%), laparoscopic adjustable gastric banding (18\%), biliopancreatic diversion (11\%), and gastric sleeve $(4 \%)$, respectively.

In the prospective observation, pre-surgery baseline evaluation (T0) was performed at hospital admission for bariatric surgery. The follow-up evaluation was performed 6 (T1) and 12 (T2) months after surgery.

\section{Biochemical and anthropometric measurements}

i) Anthropometric measures: height, weight, and WC were measured for each subject at the three time points, according to the cohort.

ii) Sex hormones: serum TT, estradiol $\left(\mathrm{E}_{2}\right)$ follicle-stimulating hormone (FSH), luteinizing hormone ( $\mathrm{LH})$, and sex hormone-binding globulin (SHBG) were measured by immunoassay (Immulite 2000, M-Medical System, Milan, Italy) on a sample of venous blood drawn in the morning. The analytical sensitivity of the assays was $0.5 \mathrm{nmol} / \mathrm{l}$ for TT, $55 \mathrm{pmol} / \mathrm{l}$ for $\mathrm{E}_{2}, 0.1 \mathrm{mIU} / \mathrm{ml}$ for FSH, $0.05 \mathrm{mIU} / \mathrm{ml}$ for $\mathrm{LH}$, and $0.02 \mathrm{nmol} / \mathrm{l}$ for SHBG. TT measurement by immunoassay has been validated by isotopic dilution-liquid chromatography-tandem mass spectrometry method for values higher than nanomolar (12). Calculated free testosterone (cFT) was calculated as demonstrated previously (13).

Table 1 Anthropometric and sex steroid parameters before bariatric surgery in the obese cohort enrolled ( $n=55$ ). Mean \pm s.D. values at baseline before surgery (T0) and at 6 (T1) and 12 months (T2) from surgery are indicated, as well as $P$ significance between the indicated time points after Student's $t$-test for paired data. Significant $P$ values are indicated in bold.

\begin{tabular}{|c|c|c|c|c|c|}
\hline & TO $(n=55)$ & $\mathbf{T 1}(n=50)$ & $P_{\mathrm{T} 1-\mathrm{To}}$ & T2 $(n=42)$ & $\boldsymbol{P}_{\mathrm{T} 2-\mathrm{TO}}$ \\
\hline Age (years) & $42.3 \pm 11.6$ & & & & \\
\hline BMI $\left(\mathrm{kg} / \mathrm{m}^{2}\right)$ & $46.6 \pm 7.4$ & $37.5 \pm 6.7$ & 0.000 & $32.2 \pm 6.8$ & 0.000 \\
\hline Weight (kg) & $148.4 \pm 26.1$ & $120.7 \pm 26.3$ & 0.000 & $101.8 \pm 24.1$ & 0.000 \\
\hline $\mathrm{WC}(\mathrm{cm})$ & $140.1 \pm 18.0$ & $121.5 \pm 17.1$ & 0.000 & $113.6 \pm 16.6$ & 0.000 \\
\hline TT (nmol/l) & $9.02 \pm 4.71$ & $14.77 \pm 6.35$ & 0.000 & $14.6 \pm 6.0$ & 0.000 \\
\hline cFT (nmol/l) & $0.224 \pm 0.132$ & $0.263 \pm 0.111$ & 0.038 & $0.265 \pm 0.097$ & 0.339 \\
\hline $\mathrm{E}_{2}(\mathrm{pmol} / \mathrm{l})$ & $139.6 \pm 49.5$ & $135.6 \pm 52.7$ & 0.347 & $126.7 \pm 44.9$ & 0.007 \\
\hline SHBG (nmol/l) & $21.7 \pm 8.9$ & $42.3 \pm 19.7$ & 0.000 & $42.2 \pm 20.0$ & 0.000 \\
\hline $\mathrm{FSH}(\mathrm{mlU} / \mathrm{ml})$ & $4.03 \pm 2.56$ & $5.59 \pm 3.00$ & 0.000 & $6.21 \pm 3.90$ & 0.000 \\
\hline $\mathrm{LH}(\mathrm{mlU} / \mathrm{ml})$ & $3.09 \pm 1.96$ & $4.34 \pm 2.14$ & 0.000 & $4.47 \pm 2.60$ & 0.001 \\
\hline
\end{tabular}




\section{Statistical analysis}

Patients were included in the analysis when evaluations at baseline and at least one follow-up were available. Parametric distribution of data was evaluated by KolmogorovSmirnov test. Data have been expressed as mean \pm s.D. Correlations were performed using Spearman's analysis. Student's $t$-test was used for comparisons of two sets of paired or independent data, as appropriate. A $P<0.05$ value was used for statistical significance. Stepwise multiple linear regression was applied for multivariate analysis. All statistical analyses were performed on SPSS 20.0 for Windows (Statistical Package for the Social Sciences).

\section{Results}

Among the 58 morbidly obese patients initially enrolled, 55 were available for analysis ( $n=55,1.2 \%$ dropout), having clinical data and blood sampling before surgery (T0, $n=55)$ and at 6 (T1, $n=50,10 \%$ dropout) and/or 12 (T2, $n=42,24 \%$ dropout) months. In the sample analyzed, the mean age was $42.3 \pm 11.6$ years, and mean BMI was $46.6 \pm 7.4 \mathrm{~kg} / \mathrm{m}^{2}$. Among those patients, 37, 10, 6, and 2, underwent Roux-en-Y gastric bypass (67\%), laparoscopic adjustable gastric banding (18\%), biliopancreatic diversion (11\%), and gastric sleeve (4\%), respectively. Six months after bariatric surgery, the decrease in adiposity (reduction in BMI, weight, and WC) was associated with a significant increase in TT and SHBG and decrease in estradiol $\left(\mathrm{E}_{2}\right)$ levels, which were further maintained at 12 months (Table 1). cFT significantly increased at 6 months, but the significance was lost at 12 months (Table 1). Gonadotropins (FSH and LH) increased, although not significantly, at both time points. When patients were stratified according to the gonadal status at baseline, in hypogonadal $(n=29)$ and eugonadal $(n=26)$ subjects (cutoff TT $=8 \mathrm{nmol} / \mathrm{l}$ ), a significant increase vs baseline was observed in both TT and cFT (Table 2), but not in $\mathrm{E}_{2}$ (Table 2), SHBG, and gonadotropins, only in the hypogonadal group (Fig. 1). Differences between hypogonadal and eugonadal patients in variations from baseline were significant only for $\Delta \mathrm{TT}$ and $\Delta \mathrm{cFT}$. No significant differences between the two groups were observed in either BMI or weight changes, but the mean variation in WC was significantly higher in hypogonadal men at 12 months (Table 2). Receiver operating characteristics (ROC) curve analysis was used to confirm the ability of the hypogonadal state (TT $<8 \mathrm{nmol} / \mathrm{l}$ ) to predict the WC reduction at 12 months (Fig. 2). Hypogonadal condition (TT $<8 \mathrm{nmol} / \mathrm{l})$, in fact, identified a WC decrease $\geq 23 \mathrm{~cm}$,

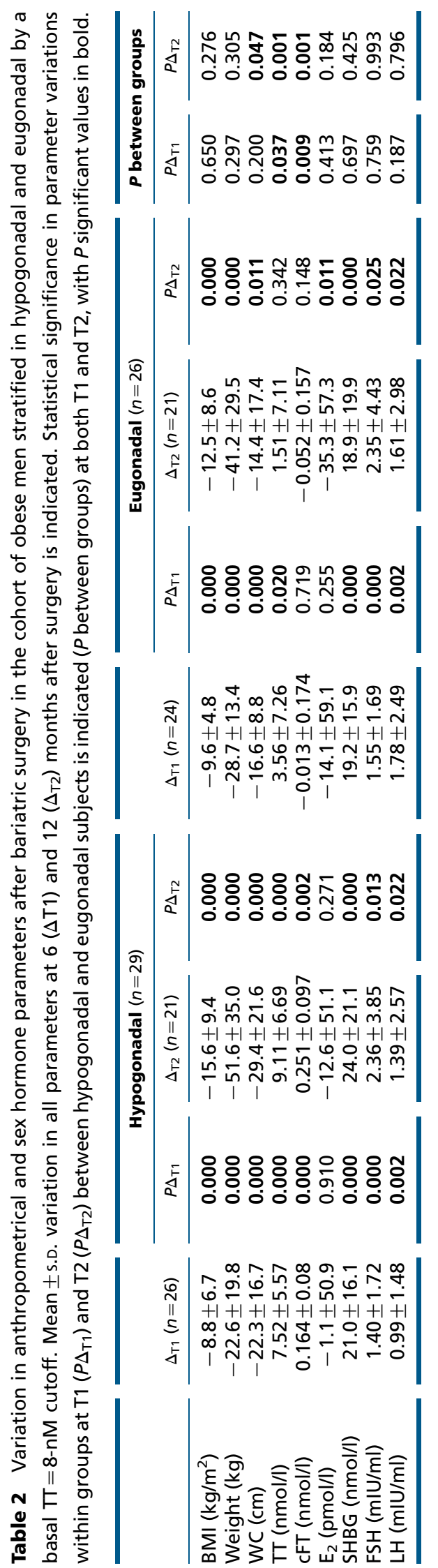



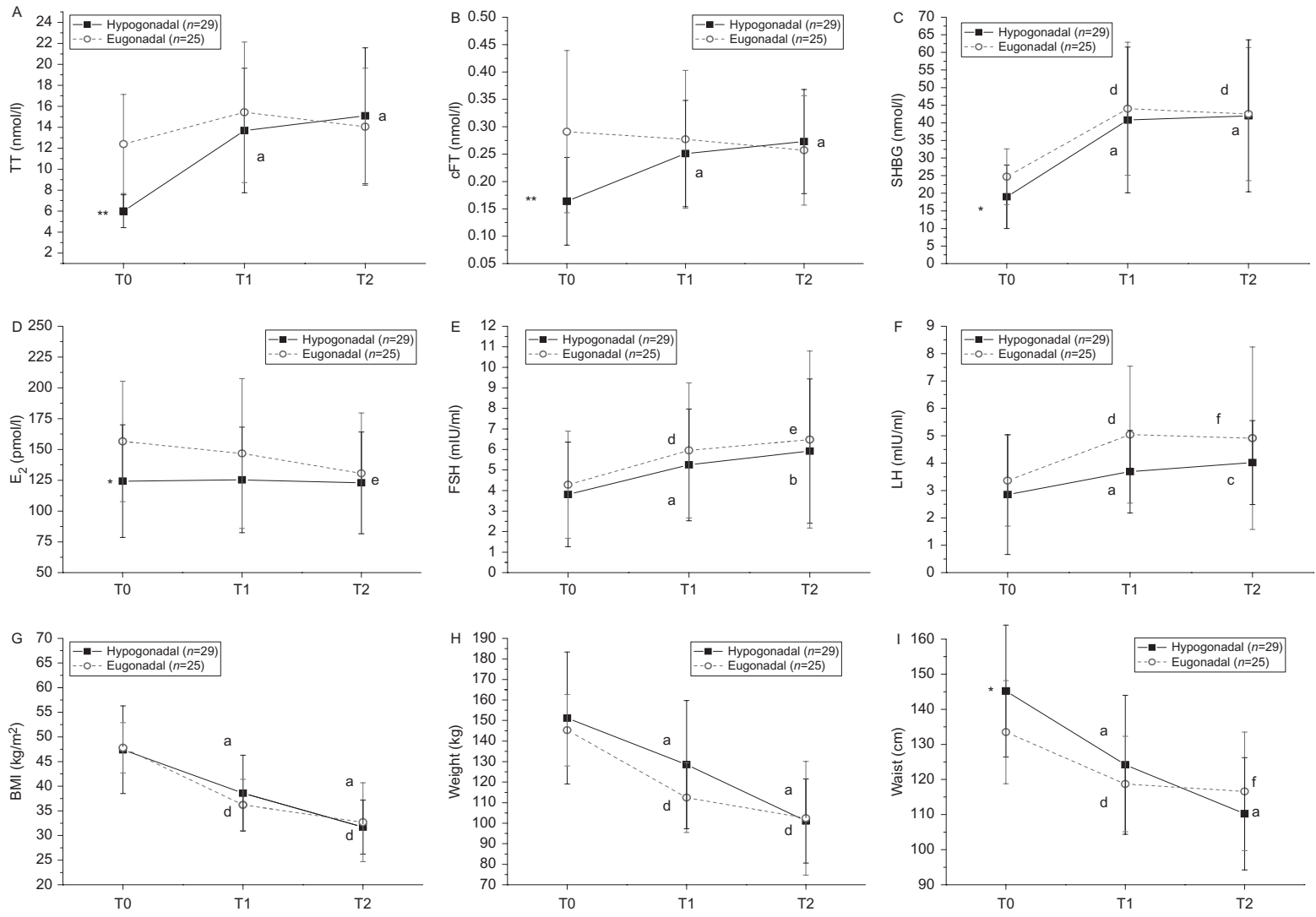

\section{Figure 1}

Variation in anthropometric and sex hormone parameters after bariatric surgery in the cohort of morbidly obese men stratified in hypogonadal and eugonadal by a basal TT $=8 \mathrm{nmol} / \mathrm{l}$ cut-off. Mean \pm s.D. values of all parameters (A: TT, B: CFT, C: SHBG, D: $E_{2}$, E: FSH, F: LH, G: BMI, H: weight, I: waist) at baseline before

with an accuracy (area under the ROC curve) of 71.2 \pm $9.9 \%(P<0.05)$, with a $67 \%$ sensitivity and $71 \%$ specificity.

$\mathrm{E}_{2}$ significantly decreased at 12 months, only in the eugonadal group (Fig. 1 and Table 2).

Bariatric surgery resulted in a complete recovery from HG (TT $<8 \mathrm{nmol} / \mathrm{l})$ in $93 \%$ of the hypogonadal subjects (27/29), already starting at 6 months from surgery (Fig. 1). No statistically significant differences were evident for baseline parameters between the two groups (BMI, weight, and gonadotropins) but for WC $(P<0.05)$, TT and cFT $(P<0.0001)$, SHBG, and $\mathrm{E}_{2}(P<0.05$; Fig. 1 and Table 2$)$. The distribution of the type of bariatric surgery was similar between hypogonadal and eugonadal groups (data not shown), and the prevalence of T2DM was $5 / 29=17 \%$ and $2 / 26=8 \%$, respectively, with no significant differences. surgery (T0) and at 6 (T1) and 12 (T2) months of follow-up. Statistical significance: ${ }^{*} P<0.05$ and ${ }^{*} P P<0.0001$ between hypogonadal and eugonadal at T0; vs T0 in each group: ${ }^{a}$ and ${ }^{\mathrm{d}} P<0.0001,{ }^{\mathrm{b}}$ and ${ }^{\mathrm{e}} P<0.01,{ }^{\mathrm{c}}$ and ${ }^{\mathrm{f}} P<0.05$, in hypogonadal and eugonadal respectively.

A strong positive correlation was found at baseline between BMI and WC in hypogonadal subjects $(r=0.811$, $P<0.0001, n=29$ ), which was still significant but weaker in eugonadal subjects $(r=0.465, P=0.045, n=26)$. Conversely, the correlation was statistically significant between $\triangle \mathrm{BMI}$ and $\triangle \mathrm{WC}$ at 12 months from surgery in hypogonadal ( $\left.r=0.864, R^{2}=0.747, P<0.0001, n=21\right)$ subjects, but not in eugonadal $\left(r=0.412, R^{2}=0.169, P=0.144, n=21\right)$ subjects. The relationship between 12-month $\triangle \mathrm{BMI}(x)$ and $\Delta \mathrm{WC}(y)$ was $y=2.132 x+0.858$ and $y=0.759 x-4.556$ in hypogonadal and eugonadal subjects respectively.

Among all parameters considered to define adiposity (BMI, weight, WC), only WC at baseline showed a statistically significant correlation with androgens ( $r=-0.375, P=0.013$ and $r=-0.411, P=0.006$ for TT and cFT respectively), but not with $\mathrm{E}_{2}$, SHBG, and gonadotropin 


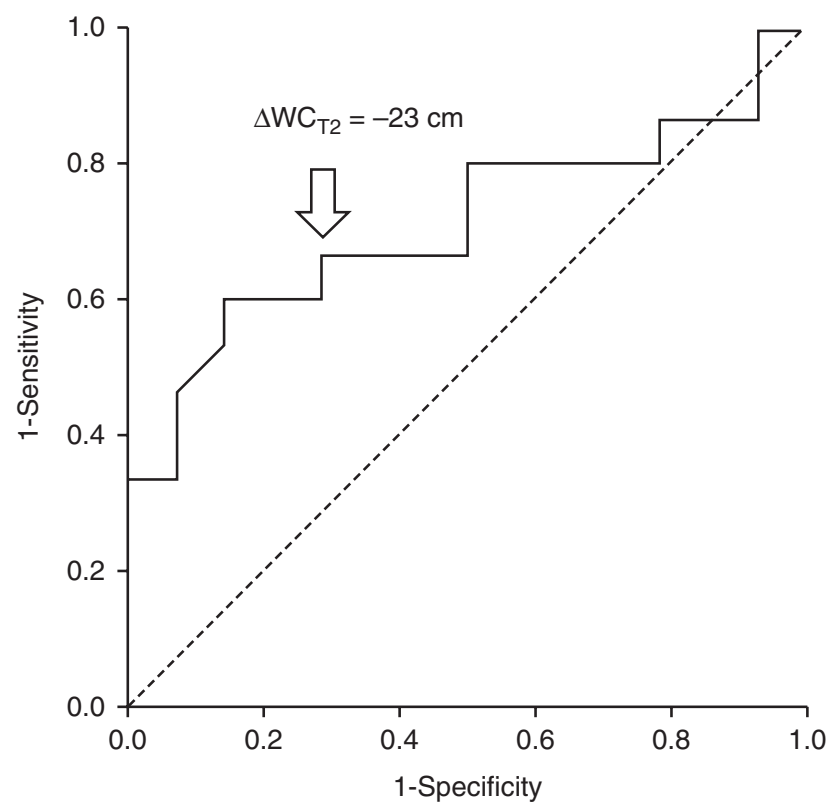

\section{Figure 2}

Hypogonadal condition predicts WC reduction at 12 months from surgery. The receiver operating characteristics (ROC) curve shows that baseline hypogonadism $(T T<8 \mathrm{nmol} / \mathrm{l})$ identifies a reduction in waist circumference greater than $-23 \mathrm{~cm}$ (indicated by the arrow, $\triangle W_{\mathrm{T}_{2}}$ ) at 12 months from surgery with an accuracy (area under the ROC curve) of $71 \%(P<0.05)$, a $67 \%$ sensitivity, and $71 \%$ specificity. The ROC curve was constructed by plotting the sensitivity (true positive) vs the false positive rate (1-specificity) for different $\Delta W C$ values at 12 months from surgery.

levels (not shown). Such negative correlations remained statistically significant, even after adjustment for age as a further confounding factor (adj. $r=0.373, P=0.013$ and adj. $r=-0.400, P=0.008$ for TT and cFT respectively).

\section{Discussion}

Bariatric surgery has been extensively described to ameliorate sex hormone and SHBG levels in morbidly obese patients $(9,14,15,16,17,18,19)$, with an effect that may exceed what is predictable on the basis of weight loss (17). In this study, we demonstrate that the increase in androgens (cFT and TT) occurs only in hypogonadal patients (TT $<8 \mathrm{nmol} / \mathrm{l})$. Conversely, estrogen levels, which were significantly lower before surgery in hypogonadal individuals, significantly decreased in eugonadal patients only. Remarkably, bariatric surgery resulted in an almost complete and rapid recovery of $\mathrm{HG}$, even starting at 6 months from surgery, similarly to what was found for T2DM recovery (20).
Interestingly, baseline testosterone levels appear to moderate the effect of bariatric surgery on sex hormone recovery. The previous works reported that $\operatorname{BMI}(9,17)$ and age (18) were among the best predictors of androgen recovery after bariatric surgery. However, such cohorts of morbidly obese patients included both hypogonadal and eugonadal subjects. In this study, we show that HG is the best predictor of the decrease in WC after surgery. It can be speculated that the rapid weight loss induced by bariatric surgery eliminates disturbances in the regulation of gonadotropin and androgen secretion determined by excess adiposity, resulting in an improvement in testosterone levels in hypogonadal subjects. Conversely, in patients who remain eugonadal despite morbid obesity, feedback from appropriate levels of circulating testosterone could prevent any further increase in androgen secretion. It can also be observed that the role of $E_{2}$ in the determination of obesity-related hypogonadism could be smaller than what has been previously hypothesized $(10,21)$; in fact, $E_{2}$ levels at baseline are lower in hypogonadal subjects compared with eugonadal subjects. In addition, in eugonadal subjects, the reduction in $\mathrm{E}_{2}$ determined by surgery does not produce an increase in testosterone, whereas recovery of hypogonadism occurs without any significant increase in $\mathrm{E}_{2}$ levels.

At enrolment, WC was higher in hypogonadal than in eugonadal patients, despite a similar BMI. This confirms that WC is one of the major predictors of obesityassociated hypogonadism $(6,9)$, suggesting that abdominal fat has a greater impact on the regulation of sex hormone secretion than peripheral fat.

Interestingly, the reduction in WC in patients with hypogonadism was more pronounced than that observed for eugonadal subjects with a similar weight loss, suggesting that bariatric surgery could have a specific effect on abdominal adiposity in hypogonadal subjects. It can be speculated that the improvement in testosterone levels induced by bariatric surgery, which is greater in hypogonadal patients, contributes, to a greater extent in those subjects, to the preservation of lean mass, resulting in a greater reduction in abdominal adiposity. Unfortunately, no measures of body composition were available in this study. Further limitations were the absence of insulin sensitivity and glycemic parameters at baseline and follow-up, and the variety of surgery procedure used, which did not allow a subgroup analysis for type of intervention. Moreover, 89\% (49/55) of the patients undergoing bariatric surgery had BMI $\geq 40 \mathrm{~kg} / \mathrm{m}^{2}$. Further studies carried out on subjects with BMI between 35 and $40 \mathrm{~kg} / \mathrm{m}^{2}$ are necessary to evaluate whether 
hypogonadism could be considered as a novel comorbidity to be considered for bariatric intervention.

In conclusion, recovery from obesity-associated hypogonadism is one of the positive clinical outcomes of bariatric surgery in morbidly obese men. In addition, male hypogonadism appears to be a predictor of reduction in WC after surgery.

\section{Declaration of interest}

Dr E Mannucci has received: consultancy fees from Novo Nordisk, Eli Lilly, AstraZeneca, and GSK; speaking fees from Novo Nordisk, Eli Lilly, AstraZeneca, and Sanofi; research grants from Novo Nordisk, Eli Lilly, and AstraZeneca. Prof. M Maggi has received consultancy fees or research grants from Eli Lilly, Bayer, and Prostrakan. The other authors have no conflicts to declare.

\section{Funding}

This study was supported by an unrestricted research grant by the Italian Ministry of University and Research (PRIN 2011 prot. 2010C8ERKX).

\section{References}

1 Finucane MM, Stevens GA, Cowan MJ, Danaei G, Lin JK, Paciorek CJ, Singh GM, Gutierrez HR, Lu Y, Bahalim AN et al. National, regional, and global trends in body-mass index since 1980: systematic analysis of health examination surveys and epidemiological studies with 960 country-years and 91 million participants. Lancet 2011377 557-567. (doi:10.1016/S0140-6736(10)62037-5)

2 Guidelines for Clinical Application of Laparoscopic Bariatric Surgery SAGES Guidelines Committee endorsed by the ASMBS. OSAGES 2008.

3 Van Gaal LF \& De Block CE. Bariatric surgery to treat type 2 diabetes: what is the recent evidence? Current Opinion in Endocrinology, Diabetes and Obesity 201219 352-358. (doi:10.1097/MED.0b013e328357f0e0)

4 Ricci C, Gaeta M, Rausa E, Macchitella Y \& Bonavina L. Early impact of bariatric surgery on type II diabetes, hypertension, and hyperlipidemia: a systematic review, meta-analysis and meta-regression on 6,587 patients. Obesity Surgery 201424 522-528. (doi:10.1007/s11695013-1121-x)

5 Tajar A, Forti G, O’Neill TW, Lee DM, Silman AJ, Finn JD, Bartfai G, Boonen S, Casanueva FF, Giwercman A et al. Characteristics of secondary, primary, and compensated hypogonadism in aging men: evidence from the European Male Ageing Study. Journal of Clinical Endocrinology and Metabolism 201095 1810-1818. (doi:10.1210/jc. 2009-1796) (doi:10.1210/jc.2009-1796)

6 Corona G, Rastrelli G, Morelli A, Vignozzi L, Mannucci E \& Maggi M. Hypogonadism and metabolic syndrome. Journal of Endocrinological Investigation 201134 557-567. (doi:10.1007/BF03347072)

8 Araujo AB, Dixon JM, Suarez EA, Murad MH, Guey LT \& Wittert GA. Clinical review: endogenous testosterone and mortality in men: a systematic review and meta-analysis. Journal of Clinical Endocrinology and Metabolism 201196 3007-3019. (doi:10.1210/jc.2011-1137)

7 Saad F, Aversa A, Isidori AM \& Gooren LJ. Testosterone as potential effective therapy in treatment of obesity in men with testosterone deficiency: a review. Current Diabetes Reviews 20128 131-143. (doi:10. 2174/157339912799424573)

9 Corona G, Rastrelli G, Monami M, Saad F, Luconi M, Lucchese M, Facchiano E, Sforza A, Forti G, Mannucci E et al. Body weight loss reverts obesity-associated hypogonadotropic hypogonadism: a systematic review and meta-analysis. European Journal of Endocrinology 2013168 829-843. (doi:10.1530/EJE-12-0955)

10 Michalakis K, Goulis DG, Vazaiou A, Mintziori G, Polymeris A \& Abrahamian-Michalakis A. Obesity in the ageing man. Metabolism 2013 62 1341-1349. (doi:10.1016/j.metabol.2013.05.019)

11 O'Reilly MW, House PJ \& Tomlinson JW. Understanding androgen action in adipose tissue. Journal of Steroid Biochemical Molecular Biology 2014 143C $277-284$.

12 Huhtaniemi IT, Tajar A, Lee DM, O'Neill TW, Finn JD, Bartfai G, Boonen S, Casanueva FF, Giwercman A, Han TS et al. Comparison of serum testosterone and estradiol measurements in 3174 European men using platform immunoassay and mass spectrometry; relevance for the diagnostics in aging men. European Journal of Endocrinology $2012 \mathbf{6 6}$ 983-991. (doi:10.1530/EJE-11-1051)

13 Vermeulen A, Verdonck L \& Kaufman JM. A critical evaluation of simple methods for the estimation of free testosterone in serum. Journal of Clinical Endocrinology and Metabolism 199984 3666-3672. (doi:10.1210/jcem.84.10.6079)

14 Hammoud A, Gibson M, Hunt SC, Adams TD, Carrell DT, Kolotkin RL \& Meikle AW. Effect of Roux-en-Y gastric bypass surgery on the sex steroids and quality of life in obese men. Journal of Clinical Endocrinology and Metabolism 200994 1329-1332. (doi:10.1210/jc.2008-1598)

15 Alagna S, Cossu ML, Gallo P, Tilocca PL, Pileri P, Alagna G, Maninchedda P, Sini AL, Pilo L, Rovasio PP et al. Biliopancreatic diversion: long-term effects on gonadal function in severely obese men. Surgery for Obesity and Related Diseases 20062 82-86. (doi:10.1016/j. soard.2006.01.005)

16 Pellitero S, Olaizola I, Alastrue A, Martínez E, Granada ML, Balibrea JM, Moreno P, Serra A, Navarro-Díaz M, Romero R et al. Hypogonadotropic hypogonadism in morbidly obese males is reversed after bariatric surgery. Obesity Surgery 201222 1835-1842. (doi:10.1007/s11695-012-0734-9)

17 Luconi M, Samavat J, Seghieri G, Iannuzzi G, Lucchese M, Rotella C, Forti G, Maggi M \& Mannucci E. Determinants of testosterone recovery after bariatric surgery: Is it only a matter of reduction of body mass index? Fertility and Sterility 201399 1872-1879. (doi:10.1016/j. fertnstert.2013.02.039)

18 Facchiano E, Scaringi S, Veltri M, Samavat J, Maggi M, Forti G, Luconi M $\&$ Lucchese M. Age as a predictive factor of testosterone improvement in male patients after bariatric surgery: preliminary results of a monocentric prospective study. Obesity Surgery 201323 167-172. (doi:10.1007/s11695-012-0753-6)

19 Reis LO, Favaro WJ, Barreiro GC, de Oliveira LC, Chaim EA, Fregonesi A $\&$ Ferreira U. Erectile dysfunction and hormonal imbalance in morbidly obese male is reversed after gastric bypass surgery: a prospective randomized controller trial. International Journal of Andrology 201033 736-744. (doi:10.1111/j.1365-2605.2009.01017.x)

20 Halperin F, Ding SA, Simonson DC, Panosian J, Goebel-Fabbri A, Wewalka M, Hamdy O, Abrahamson M, Clancy K, Foster K et al. Rouxen-Y gastric bypass surgery or lifestyle with intensive medical management in patients with type 2 diabetes: feasibility and 1-year results of a randomized clinical trial. JAMA Surgery 2014149 716-726. (doi:10.1001/jamasurg.2014.514)

21 Strain GW, Zumoff B, Miller LK, Rosner W, Levit C, Kalin M, Hershcopf RJ $\&$ Rosenfeld RS. Effect of massive weight loss on hypothalamic-pituitarygonadal function in obese men. Journal of Clinical Endocrinology and Metabolism 198866 1019-1023. (doi:10.1210/jcem-66-5-1019)
Received 16 July 2014

Accepted 6 August 2014 